\title{
Spatio-temporal missing data reconstruction in satellite displacement measurement time series
}

\author{
Alexandre Hippert-Ferrer ${ }^{1 \dagger}$, Yajing Yan ${ }^{1}$, Philippe Bolon ${ }^{1}$, Romain Millan ${ }^{2}$ \\ ${ }^{1}$ Laboratoire d'Informatique, Systèmes, Traitement de l'Information et la Connaissance (LISTIC), Annecy, France \\ ${ }^{2}$ Institut des Géosciences de l'Environnement (IGE), Université Grenoble Alpes, CNRS, Grenoble, France \\ † Correspondence to: alexandre.hippert-ferrer@univ-smb.fr
}

Thursday, May 7

LISTIC
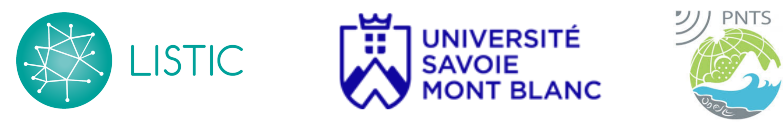


\section{Introduction}

- Missing data is a frequent issue in displacement time series in both space and time.

- It can prevent the full understanding of the physical phenomena under observation.

- Causes : rapid surface changes, missing image, technical limitations.
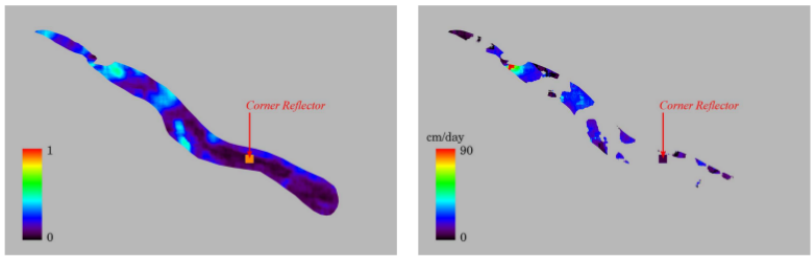

Argentière glacier, offset tracking of TerraSAR-X in Summer 2010 (Fallourd et al., 2011)

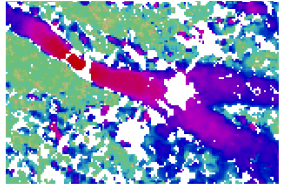

Surface velocity over Fox Glacier, offset tracking of Sentinel-2 images in February 2018 (Millan et al., 2019)

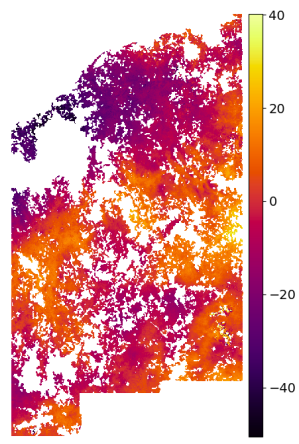

Slow slip event

(interferometry), Mexico (Maubant et al., 2020)

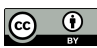


Motivation of the study

Handling missing data in displacement time series

- Classical approach : spatial or temporal interpolation

- Not exploited (yet) : spatio-temporal information

$\rightarrow$ Manage spatio-temporal missing data in time series $\leftarrow$

Objective : propose a statistical gap-filling method addressing

1. Randomness and possible spatial, temporal and spatio-temporal correlation of

- Noise

- Missing data

2. Complex displacement behaviors (mixed frequencies)

3. Small time series 


\section{Extended EM-EOF}

$\rightarrow$ Extension of the EM-EOF method (Hippert et al., 2019, 2020) [3, 4]

Key features of the extended EM-EOF method :

- Low rank structure of the sample spatio-temporal covariance matrix.

- Displacement signal and noise decomposed in empirical orthogonal functions (EOFs).

- Reconstruction with an appropriate initialization of missing values.

- Expectation-Maximization (EM)-type algorithm for resolution. 


\section{Data representation}

- Let $\boldsymbol{X}_{t}=\left\{x_{i j}(t)\right\}_{1 \leq i \leq P_{X}, 1 \leq j \leq P_{y}}$ be a spatial grid observed at time $t=1, \ldots, N$.

- Some elements of $\boldsymbol{X}_{t}$ are missing.

All $\boldsymbol{X}_{t}$ are stacked into a spatio-temporal data matrix $\boldsymbol{Y}=\left(\boldsymbol{X}_{1}, \boldsymbol{X}_{2}, \ldots, \boldsymbol{X}_{N}\right)$.

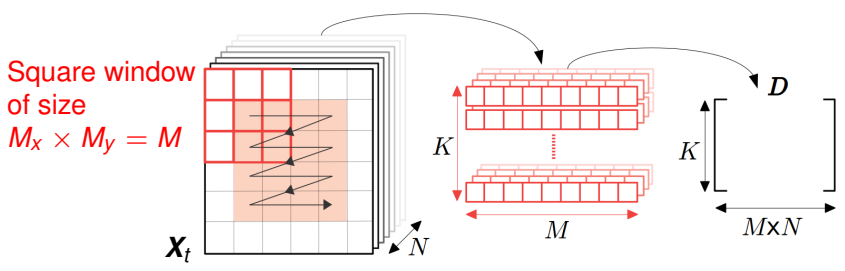

- Each $\boldsymbol{X}_{t}$ is augmented into a Hankel-block Hankel $(\mathrm{HbH})$ matrix $\boldsymbol{D}_{t}$ of size $K \times M=K_{x} K_{y} \times M_{x} M_{y}$, with $K_{x}=\left(P_{x}-M_{x}+1\right), K_{y}=\left(P_{y}-M_{y}+1\right)$.

All $\boldsymbol{D}_{t}$ is stacked into a spatio-temporal matrix $\mathcal{D}$ of size $(K \times N M)$, that is $\mathcal{D}=\left(\boldsymbol{D}_{1}, \boldsymbol{D}_{2}, \ldots, \boldsymbol{D}_{N}\right)$. 


\section{Covariance estimation and decomposition}

- Sample spatio-temporal covariance is estimated :

$$
\hat{\mathbf{C}}=\frac{1}{K} \mathcal{D}^{T} \mathcal{D}
$$

- The eigenvalue decomposition (EVD) of matrix $\hat{\mathbf{C}}$ yields to :

$$
\hat{\mathbf{C}} \stackrel{\mathrm{EVD}}{=} \sum_{i=1}^{N M} \lambda_{i} \boldsymbol{u}_{i} \boldsymbol{u}_{i}^{T}
$$

Vectors $\boldsymbol{u}_{i}$ are the NM EOFs modes of matrix $\mathcal{D}$. First modes capture the main spatio-temporal dynamical behavior of the signal, others represent perturbations.

- Reconstruction with an optimal number of EOF modes $R \ll N M$ is obtained as

$$
\hat{\mathcal{D}}=\boldsymbol{A}_{R} \boldsymbol{U}_{R}
$$

$\boldsymbol{A}$ is the matrix of principal components, which are the projection of $\mathcal{D}$ on each EEOF $\boldsymbol{u}$.

How do we find $R$ ?

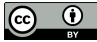




\section{Selection of the optimal number of EOF modes}

1. Root-mean-square error (cross-RMSE) on cross-validated data $\mathcal{Y} \in \mathbf{Y}$ :

$$
\frac{1}{M N}\left\|\hat{\mathcal{Y}}_{k}-\mathcal{Y}\right\|_{2}
$$

- Requires no a priori information

2. Confidence index associated with each eigenvalue of $\mathcal{D}$ :

$$
\mathcal{C}_{k}=\frac{\max \left(\Gamma_{k}\right)-\Gamma_{k}}{\max \left(\Gamma_{k}\right)-\min \left(\Gamma_{k}\right)} \quad k=1, \ldots, N M
$$

with $\Gamma_{k}=\log \left(\frac{\Delta \lambda_{k}}{\lambda_{j}-\lambda_{k}}\right)$.

- Investigation of eigenvalue degeneracy, which is linked to their uncertainties $\frac{\Delta \lambda_{k}}{\lambda_{j}-\lambda_{k}}$.

- Over-estimation of EOF modes is addressed by building metric $\mathcal{C}_{k}$. 


\section{General principle of the method}

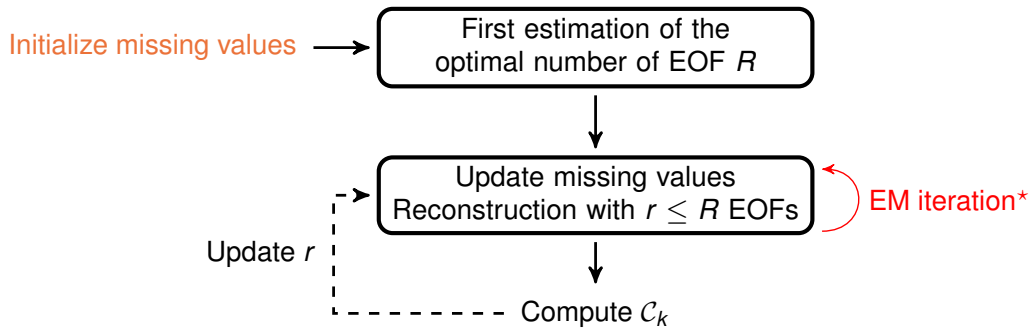

$\star$ For a fixed number of EOF modes, cross-RMSE is computed until it converges.

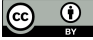




\section{Determination of the spatial lag $M$ for spatio-temporal covariance construction}

- Trade-off between the amount of information extracted in the window (large $M$ ) and the number of repetitions of the window within each image (small $M$ ).

- Upper limit based on covariance estimation theory : $M<P / 6$

- Lower limit:

We use the spatial decorrelation decay $\tau$ defined as :

$$
\tau=-\frac{\Delta P}{\log r}
$$

$r$ : lag-one auto-correlation

$\Delta P$ : spatial sampling rate, here 1 pixel.

$M$ can be approximated by $M \simeq P / \tau$ (Ghil et al., 2002) [1] which gives $M>P / 20$ with $r<0.95$. 


\section{Surface velocities on Fox Glacier, New Zealand}

\begin{tabular}{|l|l|l|l|l|}
\hline Period & Platform & Data type & Time series size & {$[\mathrm{min}, \mathrm{max}] \%$ missing } \\
\hline $02 / 2018-09 / 2018$ & Sentinel-2 & Offset tracking & 12 & {$[10,60] \%$} \\
\hline
\end{tabular}

Time series description.

- Surface velocities computed from the study of (Millan et al., 2019) [5].
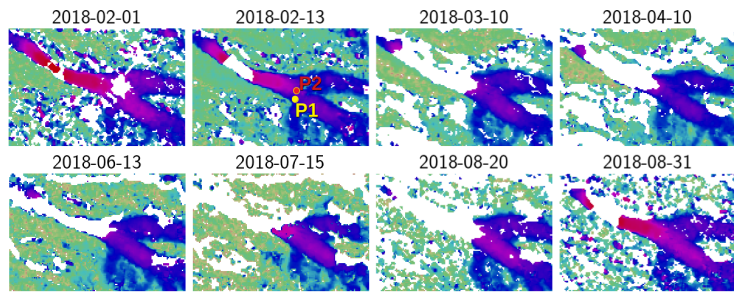

2018-05-05

2018-08-31

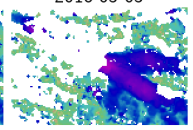

2018-05-16

2018-08-20

2018-09-10
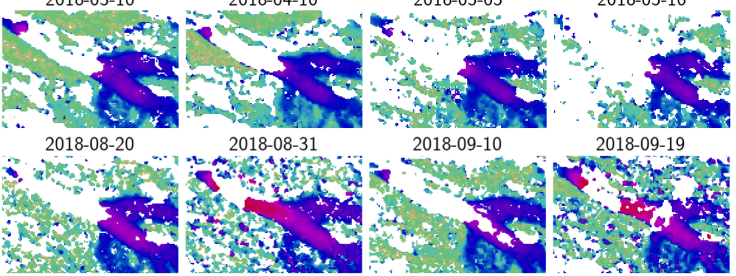

2018-09-19
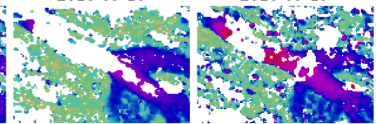

Surface velocities (m/year) on Fox Glacier. P1 and P2 locations are selected for temporal evolution analysis. 


\section{Selection of the optimal number of EOF modes}

- Optimal number of EOF modes (13) corresponds to a peak in $\mathcal{C}_{k}$ which coincides with a break in the eigenvalue spectrum.

- Eigenvalues multiplets are kept in the reconstructed data.

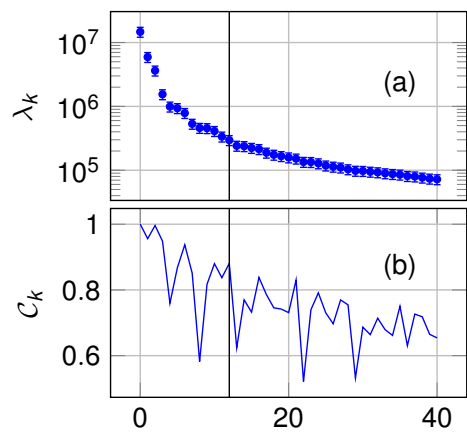

Eigenvalue number

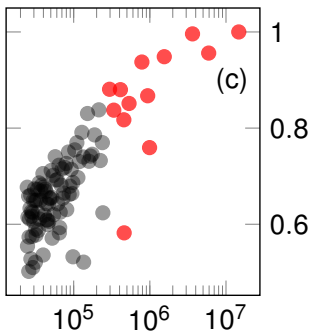

$\lambda_{k}$

$$
\mathcal{C}_{k}=\frac{\max \left(\Gamma_{k}\right)-\Gamma_{k}}{\max \left(\Gamma_{k}\right)-\min \left(\Gamma_{k}\right)}
$$$$
\Gamma_{k}=\log \left(\frac{\Delta \lambda_{k}}{\lambda_{j}-\lambda_{k}}\right)
$$ 


\section{Conclusion}

- Extension of the EM-EOF method to impute spatio-temporal missing values.

- Can handle small time series with high incompleteness

- Extraction of the displacement signal from heterogeneous perturbations (noise)

- Robust selection of the optimal number of EOF modes based on :

- Iterative computation of the cross-validation error

- Confidence metric based on eigenvalue uncertainties to address potential over-estimation due to eigenvalue degeneracy

- A range of spatial lag $M$ is provided

- Limitations : potential edge effect due to spatial square window.

Perspective : Use a shaped window (adaptive spatial lag) instead of a square window. 


\section{Bibliography}

\section{Thank you for your attention.}

[1] M. Ghil, M. Allen, M. D. Dettinger, K. Ide, D. Kondrashov, M. Mann, A. Robertson, A. Saunders, Y. Tian, F. Varadi, and P. Yiou. Advanced spectral methods for climatic time series. Review of Geophysics, 40, $1: 1-41,2002$.

[2] N. Golyandina and K. Usevich. 2d-extension of singular spectrum analysis : Algorithm and elements of theory. Matrix Methods: Theory, Algorithms and Applications, pages 449-473, 2010.

[3] A. Hippert-Ferrer, Y. Yan, and P. Bolon. Gap-filling based on iterative EOF analysis of temporal covariance : application to InSAR displacement time series. In IGARSS, pages 262-265, 2019.

[4] A. Hippert-Ferrer, Y. Yan, and P. Bolon. EM-EOF : gap-filling in incomplete SAR displacement time series. IEEE Trans. Geosci. Remote Sens., in review, 2020.

[5] R. Millan, J. Mouginot, A. Rabatel, S. Jeong, D. Cusicanqui, A. Derkacheva, and M. Chekki. Mapping surface flow velocity of glaciers at regional scale using a multiple sensors approach. Remote Sensing, 11(21), 2019.

This work has been supported by the Programme National de Télédétection Spatiale (PNTS, http ://www.insu.cnrs.fr/pnts), grant PNTS-2019-11, and by the SIRGA project. 


\section{Reconstruction averaging}

Diagonal averaging, called hankelization, [2] is applied successively to each matrix $\boldsymbol{H}_{i, t}$ and to each matrix $\boldsymbol{D}_{t}$, so that we have the following averaging :

$$
\begin{aligned}
x_{i k}(t) & =\frac{1}{\# \mathcal{A}_{k}} \sum_{\left(I, I^{\prime}\right) \in \mathcal{A}_{k}} x_{I I^{\prime}}(t) \\
\boldsymbol{H}_{k, t} & =\frac{1}{\# \mathcal{B}_{k}} \sum_{\left(I, I^{\prime}\right) \in \mathcal{B}_{k}} \boldsymbol{H}_{I^{\prime}, t}
\end{aligned}
$$

with $\mathcal{A}_{k}=\left\{\left(I, I^{\prime}\right): 1 \leq I \leq K_{y}, 1 \leq I^{\prime} \leq M_{y}, I+I^{\prime}=k+1\right\}$ and $\mathcal{B}_{k}=\left\{\left(I, I^{\prime}\right): 1 \leq I \leq K_{x}, 1 \leq I^{\prime} \leq M_{x}, I+I^{\prime}=k+1\right\}$. 


\section{Confidence index and effective sample size}

North's et al. "rule of thumb" (North, 1982) to approximate the eigenvalue uncertainty :

$$
\Delta \lambda_{k} \approx \sqrt{\frac{2}{L^{*}}} \lambda_{k} \quad \Delta \boldsymbol{u}_{k} \approx \frac{\Delta \lambda_{k}}{\lambda_{j}-\lambda_{k}} \mathbf{u}_{j}
$$

with $L^{*}=N^{*} M^{*}$.

- $N^{*}=N\left[1+2 \sum_{k=1}^{N-1}\left(1-\frac{k}{N}\right) \rho(k)\right]^{-1}$ is the temporal ESS (Thiébaux, 1984)

- $M^{*}$ is the spatial ESS within each spatial window of size $M$. We estimate it by :

$$
M^{*}=M\left(1+2 \sum_{k=1}^{M}\left(1-\frac{k}{M}\right) \nu(k)\right)^{-1}
$$

Then $\Gamma_{k}=\log \left(\frac{\Delta \lambda_{k}}{\lambda_{j}-\lambda_{k}}\right)$ and $\mathcal{C}_{k}$ is computed as :

$$
\mathcal{C}_{k}=\frac{\max \left(\Gamma_{k}\right)-\Gamma_{k}}{\max \left(\Gamma_{k}\right)-\min \left(\Gamma_{k}\right)} \quad k=1, \ldots, N M
$$

